\title{
Tuberous Breast: Combined Approach to Correct Underlying Pathology and Achieve Satisfactory Aesthetic Outcome
}

IBRAHIM H. KAMEL, M.D.

The Department of Plastic \& Reconstructive Surgery, Faculty of Medicine, Ain Shams University, Cairo, Egypt

\begin{abstract}
Introduction: Tuberous breast represents one of the challenging congenital abnormality of the breast.

Aim: This study presents a new surgical technique that combines augmentation mastopexy through inframammary and periareolar incisions with concomitant fat injection to correct tuberous breast deformity and achieve a satisfactory outcome.
\end{abstract}

Patients and Methods: 10 patients with congenital tuberous breast underwent this multi-approach. Preoperative and postoperative breast measurements were obtained and compared. Follow-up ranged from 9-24 months with a mean of 12.6 months. Results were graded according to Pacifico and Kang 2007 assessment scale.

Results: Satisfactory aesthetic outcome could be obtained that was maintained during the follow-up period.

Conclusion: This multi approach shows very high satisfactory aesthetic results that can be maintained. Larger patient population and longer follow-up can validate the efficacy of the technique.

Key Words: Tuberous breast - Congenital breast anomalies - Breast ptosis - Fat injection.

\section{INTRODUCTION}

Tuberous breast syndrome was first described by Rees and Aston [1] in 1976. It is characterized by breast hypoplasia, breast tissue herniation through the enlarged areola, elevated inframammary fold, and constricted breast base; these features have a very variable range of clinical presentation.

The incidence of tuberous breast deformity is not clearly documented and variable [2,3]. Zambakos et al. [3] suggested that the actual incidence is unknown. Several classification systems had been reported to define the wide spectrum of this breast deformity [4-7].

Although the exact etiology is not clear, it is generally accepted that the tuberous breast disorder has an embryologic origin the superficial fascia of the breast is abnormal and constrict at the base of the breast and to a deficient areola resulting in reduced breast base diameter and areolar herniation [8]. A different theory states that the deficient are- olar support rather than a constricted base is the cause of the deformity [9].

Several surgical techniques have been suggested to correct deformities of tuberous breasts [10]. This wide variety of techniques reflect the surgical challenges that requires continuous evolution for the suitable surgical technique. This study presents a surgical technique that combines augmentation mastopexy through inframammary and periareolar incisions with concomitant fat injection to correct tuberous breast deformity and achieve a satisfactory aesthetic outcome.

\section{PATIENTS AND METHODS}

This study included 10 female patients (18 breasts) complaining of tuberous breast deformity in the period of 2013-2017. All patients had no associated co-morbidity. Detailed history taking and physical examination were performed including breast size, consistency, skin deficiency, suspicious masses, and nipple areola complex (NAC) position in relation to inframammary fold (IMF) and to the suprasternal notch $(\mathrm{SN})$.

Preoperative mammogram was done for all patients to exclude presence of non-palpable breast masses. The idea of this paper was approved by the hospital scientific committee. The benefits and possible drawbacks were discussed with all patients and informed consent was obtained. All patients underwent surgical treatment with combined incisions and fat grafting at a single time.

\section{Preoperative makings (Fig. 1):}

The patient was marked in the standing position. The midline, breast meridian, and the inframammary fold (IMF) were marked. The measurements of suprasternal notch to nipple (SN-N), breast base, and nipple to inframammary fold distances (N-IMF) were recorded. The distance of the future areola to suprasternal notch ranged between $19-22 \mathrm{~cm}$. the size of the new nipple-areolar complex was of marked as an average diameter of $4.5 \mathrm{~cm}$. The limits of dissection for implant accommodation were 
planned; the lateral boundary was the anterior axillary line. The medial boundary was nearly 2 to $3 \mathrm{~cm}$ lateral to the mid-sternal line. The upper limit was about $3-4 \mathrm{~cm}$ below the clavicle. The lower limit was defined by the position of the future inframammary fold; in unilateral cases we matched the contralateral side while in bilateral cases, it was about $5 \mathrm{~cm}$ below the nipple according to patient wishes and surgeon decision. The inframammary incision was marked at future crease $4 \mathrm{~cm}$ long.

\section{Surgical technique (Fig. 2):}

The inframammary incision was done first. The retromammary pocket was created using blunt and sharp dissection for application of the silicone implants. Wide dissection is used to free all the flap all around to accommodate the implant. Dissection is carried on carefully in the deficient lower areas toward the inframammary fold. In addition, this wide dissection served also to free the constricting band of the tuberous breast without the need for incisions across the glandular tissue. Sometimes, we needed to perform a limited relaxing one or two incisions across the constricting band from the retromammary space but not extending through the whole glandular tissue.

Pocket irrigation with saline containing gentamycin is done after insertion of the desired implant. The implants were textured surface, round base, and high or extra-high profile of different volumes (ranging from 220 to $330 \mathrm{ml}$ ).

The superficial fascia layer is closed first by 0 PDS or Maxon suture. The incision line is closed with 2-0 interrupted absorbable subcutaneous sutures and non absorbable 4/0 sutures for skin closure.

Next, the periareolar incision was done measuring $4.5 \mathrm{~cm}$ in diameter. The remaining enlarged areolar tissue was de-epithelialized to achieve proper position and adequate diameters of the areolas. The periareolar incision is closed in 2 layers using PDS 3/0 and nylon $4 / 0$.

After closure of the incisions, we repeat the examination of the breast for any areas that appear deficient of tissues and we mark these areas. Limited liposuction is performed mostly from the lower abdomen or trochanteric areas after injection of tumescent fluid. Fat is allowed to sink down, and the excess fluid is evacuated. We used a cannula size 3 for liposuction and infiltration. Fat is injected in the desired areas for better contouring till the desires shape is reached (ranged from 20-100cc). At the end of the procedure, a compressive non-occlusive dressing was applied. An elastic compression brassiere was used postoperative and continued for one month postoperative.
Follow-up: All patients were followed monthly within the first 3 months then every 3 months for another 12 months. Digital photos were taken before surgery and postoperatively. The aesthetic outcome was graded as excellent ( $>75 \%$ improvement), good (50/75\% improvement), and fair ( $<50 \%$ improvement) based on assessment criteria that are adopted from Pacifico \& Kang [9] scale and included:

1- Correction of deformity (corrected, mild residual deformity, uncorrected);

2- Patient satisfaction (very satisfied, satisfied, unsatisfied); and

3- Independent review by a panel of three plastic surgeons (excellent, good, poor).

We also used the Visual Analogue Scale (VAS) according to Dessy et al. [11]. Breast shape was subjectively evaluated by the patient herself one year after surgery giving a score from 1 to 10 (1=none correction of breast asymmetry, $10=$ no residual difference between breasts). Preoperative and postoperative breast measurements were also recorded in order to evaluate symmetry achievement.

The incidence of complications were also recorded including seroma, hematoma, wound dehiscence, wound infection, scarring, recurrence, loss of sensation, and implant related complications.

\section{RESULTS}

Patients' age ranged between 16-25 years (mean of 18.4 years). Patients' weight ranged from 55$69 \mathrm{~kg}$ with a mean of $56.9 \mathrm{~kg}$ and body mass index ranged from 20.6 to 27.3 with a mean of 23.1. All surgical procedures were done under general anesthesia and by the same surgical team. Followup ranged from 6 months to 24 months with mean of 11.2 months.

All patients experienced satisfactory outcome (Figs. 3-5). The treated breasts showed a near normal-sized areola, near natural shape, accepted symmetry and no residual evidence of tuberous deformity. Furthermore, it was noted that the aesthetic outcome improved by time. One patient required secondary procedure in the form of fat injection for a palpable implant in the inferior portion. Another patient required repeated fat injection for deficient contour. No patients required scar revision of both the inframammary or the periareolar scars. No other complications were recorded.

On the visual analogue scale, 6 breasts had score of 10, 10 breasts had score of 9, and 2 breasts had score of 8 with average of (9.22). No significant differences were recorded between both breasts' measurements. 

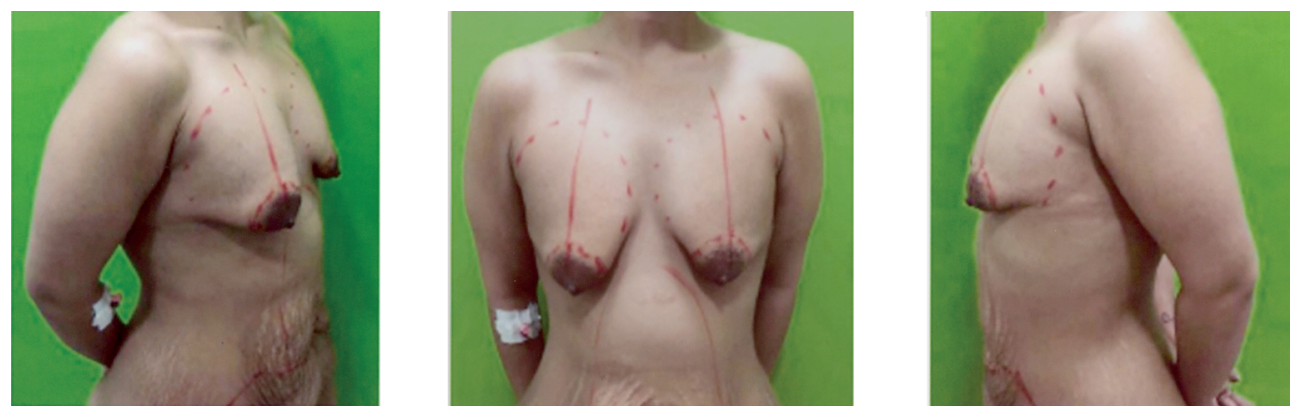

Fig. (1): Preoperative markings showing breast meridian and foot prints.
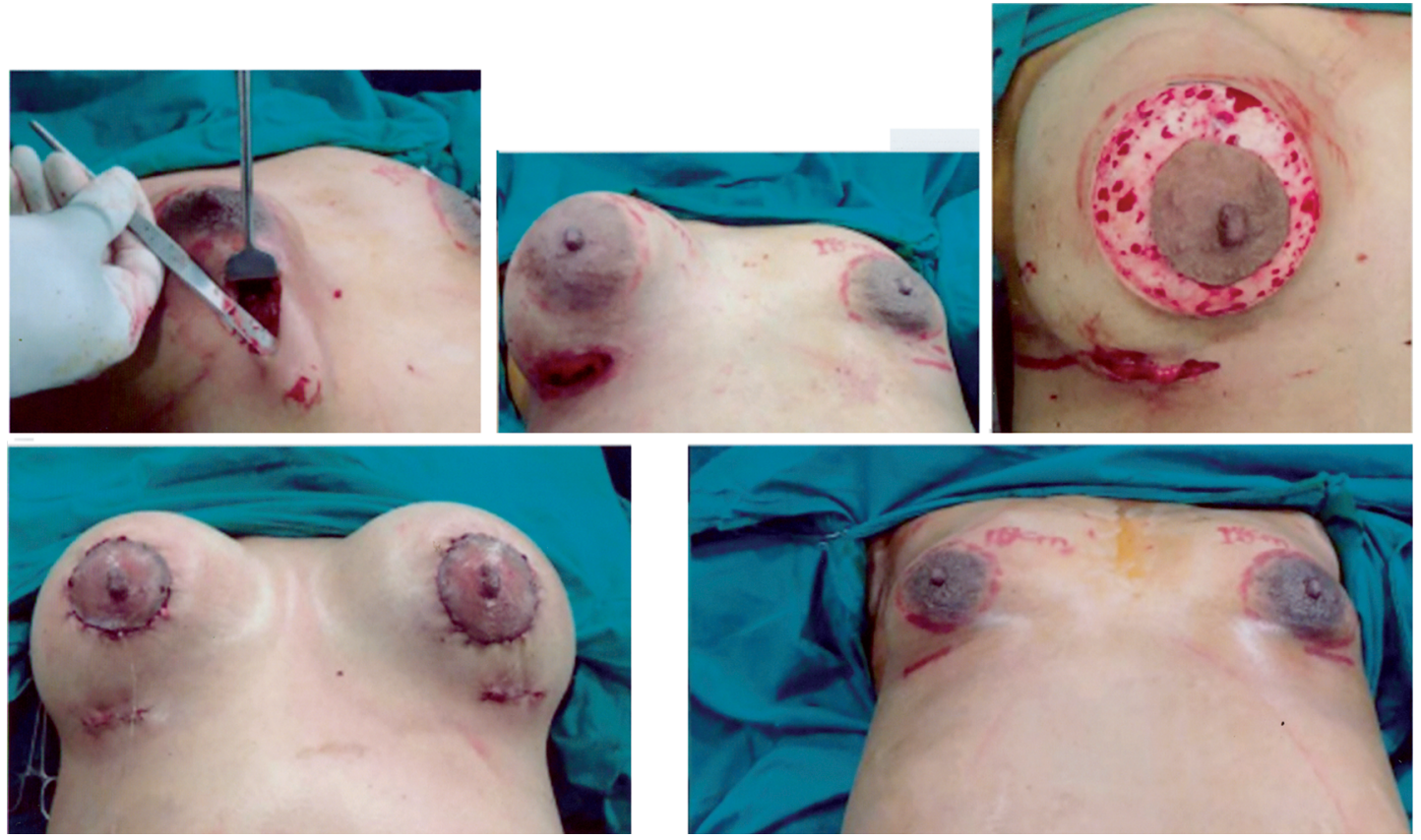

Fig. (2): (A) Inframammary incision, dissection of inframammary crease and scoring incisions of the constricting bands, (B) Isertion of mammary implant, (C\&D) Reduction of areolar size and suturing, (E) Immediate pre \& post operative result.
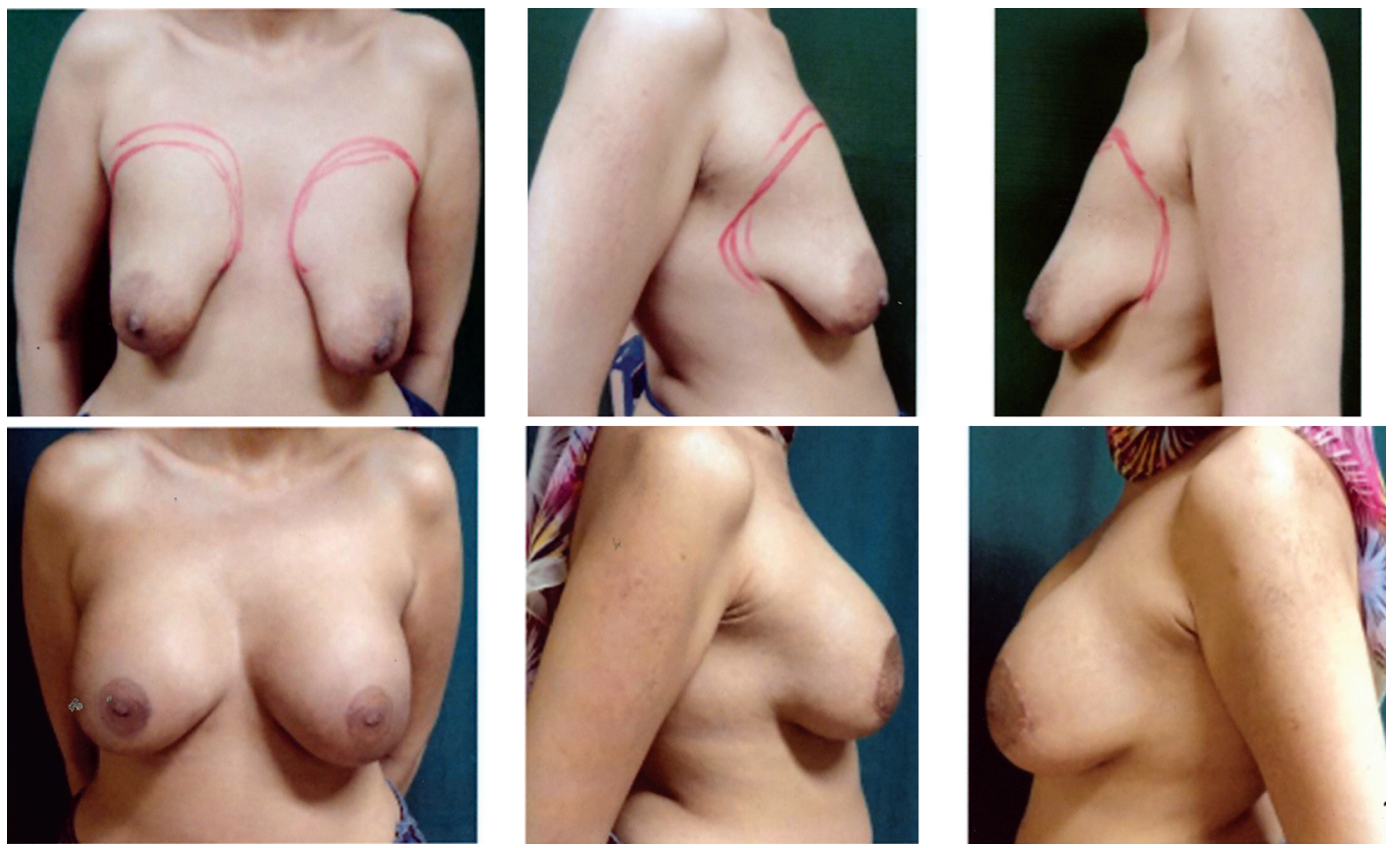

Fig. (3): Preoperative and postoperative views of 33 years patient. 

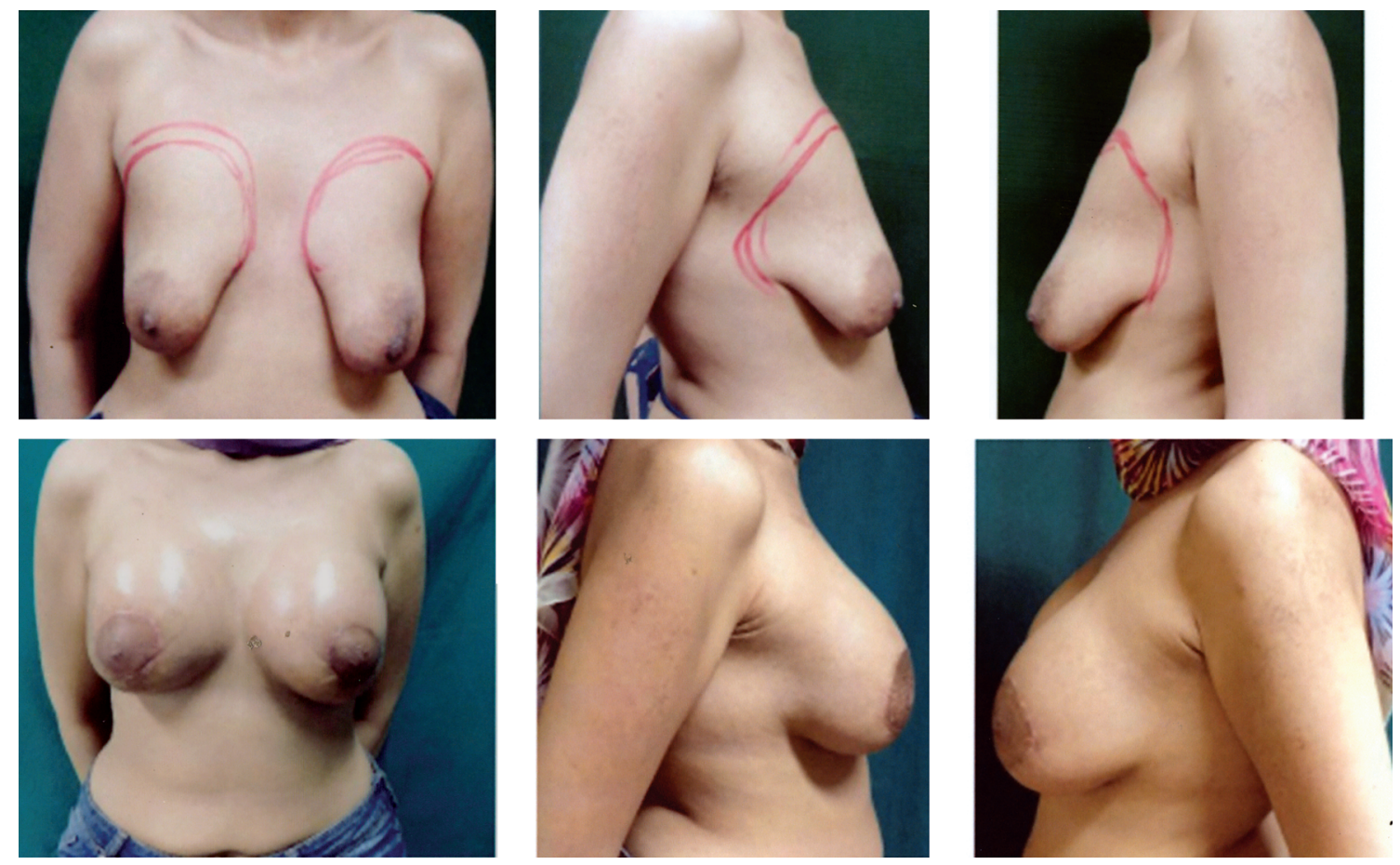

Fig. (4): Preoperative and postoperative views of 36 years patient.
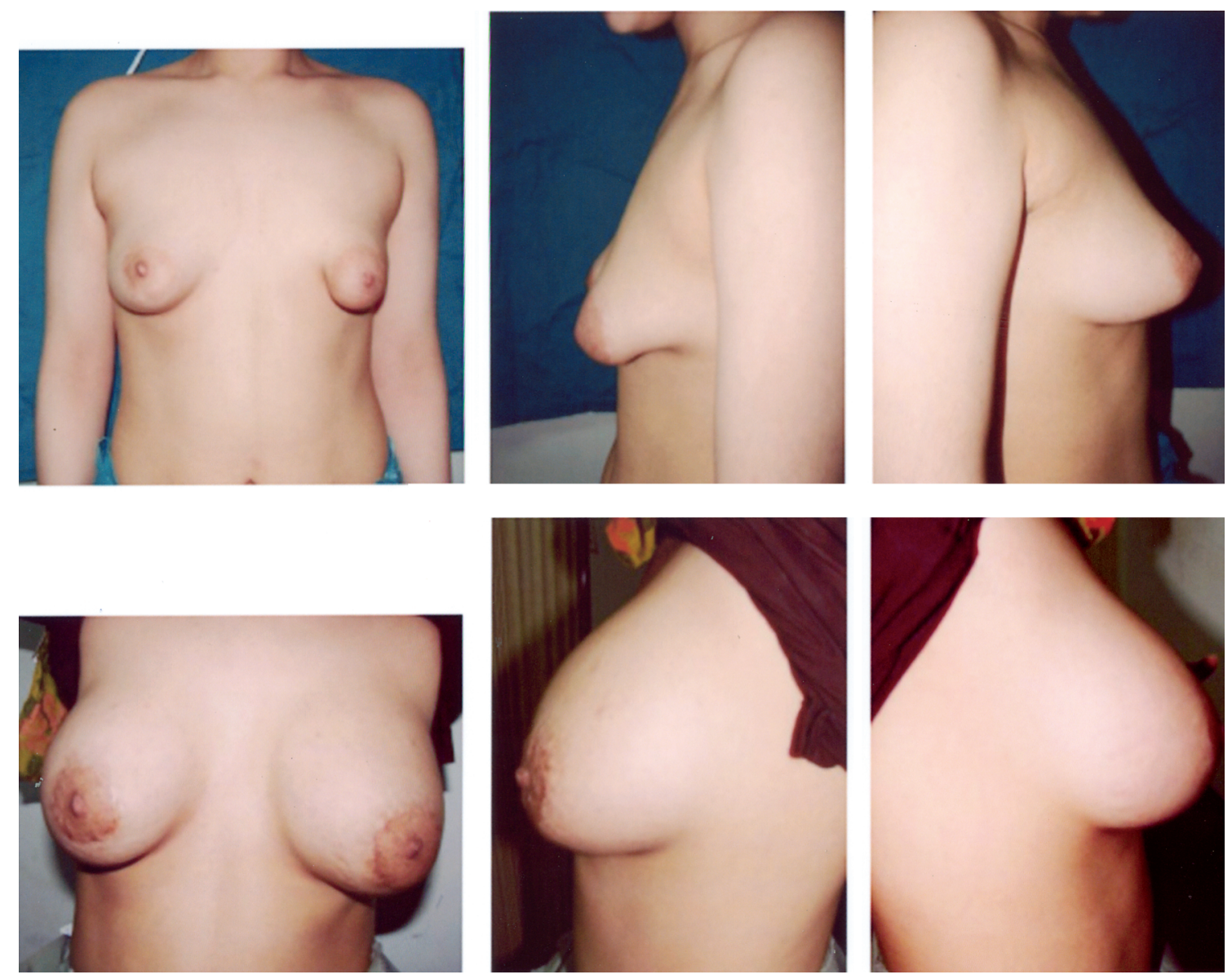

Fig. (5): Preoperative and postoperative views of 21 years patient. 


\section{DISCUSSION}

The tuberous breast deformity is a source of profound psychological concern, necessitating surgical correction to improve the aesthetic appearance [11]. The treatment of such a condition is a real surgical challenge $[\mathbf{1 2}, \mathbf{1 3}]$. The main goals are: To correct the deformity in one-stage, restore the mammary base dimensions, correct the hypoplasia of the lower quadrants, reposition the inframammary fold, reduce the areolar dimensions (correcting NAC herniation, if present), and to achieve a good symmetry [14].

The wide range of presentation, in conjunction with frequent asymmetry, poses great surgical challenges in aesthetic correction [15]. Several surgical techniques have been described which reflects the diversity of the deformity $(1,7,9,16-$ 22). In 1976, Rees and Aston first advocated radial scoring incisions at breast base [1]. Dinner and Dowden [16] performed skin incision as they thought that the constricting band is cutaneous. Ribeiro et al., identified a constrictive ring and divided it horizontally $[\mathbf{1 7}, \mathbf{1 8}]$ while Mandrekas et al. [8] divided the band vertically. Pacifico and Kang [9] believed that areolar abnormality is the underlying pathology. They described areolar reduction, subdermal undermining, and subglandular implant augmentation. Coleman and Saboeiro [19] used autologous fat injection into the subcutaneous tissues and pectoral muscle.

The periareolar approach is the most popular among most of these studies. Kolker and Collins [15] stated that periareolar approach affords the most flexibility and predictability, and they use this approach exclusively for the correction of tuberous breast deformities. Furthermore, they said that periareolar transglandular approach is ideal for facilitating the exposure to this lower pole subglandular plane, and for radially dividing the fascial constriction. They did not support the inframammary approach claming that the inframammary incision is extremely difficult to determine precisely leading to improper final scar placement.

Although periareolar incision has the benefit of minimizing incisions, surgical review was needed in up to $53 \%$ of cases to correct and enlarged periareolar scar [23]. Furthermore, the inframammary incision has the advantages such as greater technical ease in preparing the retroglandular accommodation and inclusion of the breast implant, less tissue trauma, better exposure of the surgical field, and improved control of hemostasis. Additionally, the incision is inconspicuous, which fa- cilitates establishment of the position of the new mammary ridge [10].

Teimourian and Adham [22] described the use of combined periareolar and inframammary incisions for breast implants application. They reported good results. This approach did not gain popularity and was not published again until 2012 when Neto et al. [10] used combined inframammary (for implant insertion) and periareolar incisions (for areola diameter adjustment and treatment of the fibrous ring). They concluded that this approach allows better control of hemostasis and less tissue trauma. It is also an easier technique, as it involves widespread access to the fibrotic pillars.

Although when we started our work, Neto et al. [10] work was not published yet, however, our thoughts and believes were nearby to Thiers. Inframammary approach is very easy, fast, and most surgeons are familiar with. It permits easy application and easy control of the implant. The location of the scar can be easily determined as a mirror in unilateral cases. In bilateral cases, the IMF NAC distance can be used as a guide in relation to other measurements and patient's wishes. In addition, the IMF allows wide dissection of the mammary tissues which allows division of the constricting band. If there is remaining band is present, a limited relaxing incision can be done but not throughout the entire glandular tissue. This limited incision do not disrupt glandular tissues markedly and minimizes trauma the mammary gland that can help to preserve higher possibilities for lactation and NAC sensations.

In our surgical technique, the periareolar incision is a superficial incision. Its role is mainly to reduce the diameter of enlarged areola which can be adjusted easily and more accurately after implant insertion. There is minimal tension which avoids the major drawback of postoperative enlarged areolar in peri-areolar approach.

By the end of the procedure, reassessment is carried on and the role of fat injection takes place. Fat injection is very useful and helpful for contour adjustment and asymmetry treatment. Furthermore, its safety had been established in the last decade $[\mathbf{2 4 , 2 5}$. In addition, fat injection especially in the inferior part adds more tissue and cover provides further covering for the breast implant.

Our technique is near to Neto et al. [10] but there is some technical differences. First, they start by periareolar incision while we prefer the inframammary incision first. They perform dissection from both incisions while we limit the glandular 
trauma to the inframammary approach only. They perform 4 relaxing incisions as routine while we use only 2 incisions when needed. We use only periareolar incision for areolar reduction not for dissection which permits tension free closure and avoids areolar scar widening. Lastly, we perform fat injection as an integral part of the technique while they do not.

Our technique showed very satisfactory aesthetic outcome with very high patient satisfaction. Also, the technique is very safe and did not record any major complication; only two patients required secondary procedure.

Kolker and Collins [15] reported global complication rate 7.8 percent, with capsular contracture in two breasts (3.9 percent) and malposition in two breasts (3.9 percent). Mandrekas et al. [8] in their study of 11 patients, and Pacifico and Kang (eight patients) [9] reported no complications.

In general, reported outcomes showed marked improvement and ranged from good to excellent results. Mandrekas et al. [8] reported 100\% patient satisfaction and $100 \%$ surgeon satisfaction. Pacifico and Kang [9] reported excellent outcome in $75 \%$ and good in 25\%; however, all patients reported that they were very satisfied with the outcome.

Miguel Choupina [14] recommended treatment according to the severity of the case. He proposed periareolar augmentation mastopexy for type I deformities. For type II or III breast deformity, glandular remodeling of the mammary tissue is essential. This can be performed with a glandular remodeling bringing breast tissue to the lower quadrants $[18,23,26]$.

Our technique can be used for any classification system and for any grades. Our thought is similar also to that of Neto et al. [10]; They concluded that classification of the tuberous breast into different degrees of severity seems unnecessary since the same procedure is able to achieve the same result in every case.

\section{Conclusion:}

The combined use of inframammary and periareolar approach showed highly effective as well as high safety results. The IMF approach facilities wide dissection, constricting band release, and implant insertion. The periareolar incision is used for areolar reduction minimizing breast tissue trauma and maximizing technique safety. The complementary use of fat injection adds more flexibility to the technique making individualiza- tions of the technique to be suitable for every case much easier and more effective.

\section{REFERENCES}

1- Rees T.D. and Aston S.J.: The tuberous breast. Clin. Plast. Surg., 3: 339, 1976.

2- Deluca-Pytell D.M., Piazza R.C., Holding J.C., Snyder N., Hunsicker L.M. and Phillips L.G.: The incidence of tuberous breast deformity in asymmetric and symmetric mammaplasty patients. Plast. Reconstr. Surg., 116: 1864$1899,2005$.

3- Zambacos G.J. and Mandrekas A.D.: The incidence of tuberous breast deformity in asymmetric and symmetric mammaplasty patients. Plast. Reconstr. Surg., 118: 1667, 2006.

4- Von Heimburg D., Exner K., Kruft S. and Lemperle G.: The tuberous breast deformity: Classification and treatment. Br. J. Plast. Surg., 49: 339-345, 1996.

5- Grolleau J.L., Lanfrey E., Lavigne B., Chavoin J.P. and Costagliola M.: Breast base amomalies: Treatment strategy for tuberous breasts, minor deformities, and asymmetry. Plast. Reconstr. Surg., 104: 2040-2048, 1999.

6- Costagliola M., Atiyeh B. and Rampillon F.: Tuberous breast: Revised classification and aa new hypothesis for its development. Aesthetic Plast. Surg., 37: 896-903, 2013.

7- Meara J.G., Kolker A., Bartlett G., Theile R., Mutimer K. and Holmes A.D.: Tuberous breast deformity: Principles and practice. Ann. Plast. Surg., 45: 607-611, 2000.

8- Mandrekas A.D., Zambacos G.J., Anastasopoulos A., et al.: Aesthetic reconstruction of the tuberous breast deformity. Plast. Reconstr. Surg., 112: 1099-1108, 2003.

9- Pacifico M.D. and Kang N.V.: The tuberous breast revisited. J. Plast. Reconstr. Aesthet. Surg., 60: 455-464, 2007.

10- Neto M.P., Colombo L.R., Silva D., et al.: Treatment of tuberous breast with combined incisions. Rev. Bras Cir. Plást., 27 (3): 421-7, 2012.

11- Dessy L.A., Mazzocchi M., Corrias F., Sorvillo V. and Scuderi N.: Correction of tuberous breast with small volume asymmetry by using a new adjustable implant. European Review for Medical and Pharmacological Sciences, 17: 977-983, 2013.

12- Benelli L.: Technique personnelle de plastie mammaire perareolaire: le round-block. Cah Chir., 77: 15, 1991.

13- Kneafsey B., Crawford D.S., Khoo C.T.K. and Saad M.N.: Correction of developmental breast abnormalities with a permanent expander/implant. Br. J. Plast. Surg., 49: 302, 1996.

14- Choupina M., Malheiro E., Pincho C., Ferreira A., Pinto A., Cardoso A., Reis J. and Amarante J.: Tuberous Breast: A surgical challenge. Aesth. Plast. Surg., 26: 50-53, DOI: 10.1007/s00266-001-0007-9, 2002.

15- Kolker A.R. and Collins M.S.: Tuberous breast deformity: Classification and treatment strategy for improving consistency in aesthetic correction. Plast. Reconstr. Surg., 135: 73, 2015.

16- Dinner M.I. and Dowden R.V.: The tubular/tuberous breast syndrome. Ann. Plast. Surg., 19: 414-420, 1987. 
17- Ribeiro L., Accorsi A.Jr., Buss A. and Marcal-Pessoa M.C.: Short scar correction of the tuberous breast. Clin. Plast. Surg., 29: 423-431, 2002.

18- Ribeiro L., Canzi W., Buss A. and Accorsi A.Jr.: Tuberous breast: A new approach. Plast. Reconstr. Surg., 101: 4250, 1998.

19- Coleman S.R. and Saboeiro A.P.: Fat grafting to the breast revisited: Safety and efficacy. Plast. Reconstr. Surg., 119: 775-785, 2007.

20- Panettiere P., Del Gaudio G-A., Marchetti L., Accorsi D. and Del Gaudio A.: The tuberous breast syndrome. Aesthet. Plast. Surg., 24: 445-449, 2000.

21- Gasperoni C., Salgarello M. and Gargani G.: Tubular breast deformity: A new surgical approach. Eur. J. Plast. Surg., 9: 141-145, 1987.
22- Teimourian B. and Adham M.N.: Surgical correction of the tuberous breast. Ann. Plast. Surg., 10: 190-193, 1983.

23- Argenta L.C., VanderKolk C., Friedman R.J. and Marks M.: Refinements in reconstruction of congenital breast deformities. Plast. Reconstr. Surg., 76 (1): 73-82, 1985.

24- Delay E., Sinna R., Checkaroua K., Delaporte T., Garson S. and Toussoun G.: Lipomodeling of Poland's syndrome: A new treatment of the thoracic deformity. Aesthetic Plaast. Surg., Apr., 34 (2): 218-25, 2010.

25- Coleman S. and Saboeiro A.: Fat grafting to the breast revisted: Safety and efficacy. Plast. Reconstr. Surg. Mar., 119 (3): 775-85; discussion 786-7, 2007.

26- Puckett C.L. and Concannon M.J.: Augmenting the narrowbased breast: The unfurling technique to prevent the double-bubble deformity. Aesthetic Plast. Surg., 14: 1519, 1990. 